\title{
Magnetic island merger as a mechanism for inverse magnetic energy transfer
}

\author{
Muni Zhou, ${ }^{1}$ Pallavi Bhat, ${ }^{1,2}$ Nuno F. Loureiro, ${ }^{1}$ and Dmitri A. Uzdensky ${ }^{3}$ \\ ${ }^{1}$ Plasma Science and Fusion Center, Massachusetts Institute of Technology, Cambridge, Massachusetts 02139, USA \\ ${ }^{2}$ Department of Applied Mathematics, University of Leeds, Leeds LS2 9JT, United Kingdom \\ ${ }^{3}$ Center for Integrated Plasma Studies, Physics Department, UCB-390, University of Colorado, Boulder, Colorado 80309, USA
}

(Received 8 January 2019; published 9 August 2019)

\begin{abstract}
Magnetic energy transfer from small to large scales due to successive magnetic island coalescence is investigated. A solvable analytical model is introduced and shown to correctly capture the evolution of the main quantities of interest, as borne out by direct numerical simulations. Magnetic reconnection is identified as the key mechanism enabling the inverse transfer, and setting its properties: Magnetic energy decays as $\tilde{t}^{-1}$, where $\tilde{t}$ is time normalized to the (appropriately defined) reconnection timescale, and the correlation length of the field grows as $\tilde{t}^{1 / 2}$. The magnetic energy spectrum is self-similar, and evolves as $\propto \tilde{t}^{-3 / 2} k^{-2}$, where the $k$ dependence is imparted by the formation of thin current sheets.
\end{abstract}

DOI: 10.1103/PhysRevResearch.1.012004

Introduction. The (inverse) transfer of magnetic energy from small to large spatial scales is a poorly understood plasma process of fundamental relevance to a variety of space and astrophysical environments. It may, for example, play a critical role in the origin of large-scale galactic magnetic fields [1], by enabling kinetic-scale seed fields (e.g., Weibel [2] generated) to develop spatial coherence on larger, perhaps fluid, scales [3]. Ultimately, the questions are not only whether such an inverse transfer is possible, but also how rapid and efficient it is-i.e., can an inverse transfer deliver significant amounts of magnetic energy to scales where ambient turbulence may efficiently amplify it via turbulent dynamo processes?

Similarly motivated issues arise in the context of gammaray bursts (GRBs) where one wonders if Weibel-produced fields in relativistic shocks [4] can survive long enough to explain the observed synchrotron emission [3]. In the spacephysics context, a frequently encountered question concerns the dynamical evolution of a volume-filling "sea" of flux ropes, e.g., in the solar wind and the outer heliosphere [5-8].

Past theoretical work on inverse magnetic energy transfer has mainly developed along two directions: (i) the study of magnetohydrodynamic (MHD) turbulence (e.g., Refs. [9-18]), where the inverse transfer arises from the conservation of the square vector potential in two-dimensional (2D) systems [19,20] and magnetic helicity in threedimensional (3D) systems [21,22], and (ii) the long-term evolution of Weibel-generated current filaments via their coalescence [3,23-27]. Though this problem has been widely explored, a dynamical model explaining its essential physical processes and characteristic timescale is still lacking. In this

Published by the American Physical Society under the terms of the Creative Commons Attribution 4.0 International license. Further distribution of this work must maintain attribution to the author(s) and the published article's title, journal citation, and DOI.
Rapid Communication, we build upon ideas from both of these camps to present a conceptually different picture of inverse energy transfer which essentially relies on magnetic reconnection as the enabler of such a process. Unlike previous classic work on this problem [20,28], our interpretation of this inverse transfer does not follow from any turbulence closure model, nor does it appeal to the concepts of eddy noise or eddy viscosity (and, indeed, the results we obtain cannot be explained by such concepts).

Hierarchical coalescence of magnetic islands. An analytically tractable model for inverse magnetic energy transfer is provided by a two-dimensional ensemble of magnetic islands whose evolution proceeds via their coalescence [29]. For simplicity, we adopt the incompressible resistive MHD framework, but we note that our ideas should qualitatively carry over to more advanced plasma descriptions.

We first assume that the (hierarchical) merging process occurs in discrete stages; at each stage (or generation, $n$ ), all islands are assumed identical and circular. At any given $n$th generation, a magnetic island is characterized by its radius $R_{n}$ and the total magnetic flux it encloses, $\psi_{n}$. The typical magnetic field in the island, $B_{n}=\psi_{n} / R_{n}$, and the magnetic energy it contains, $\epsilon_{n} \simeq \pi R_{n}^{2} B_{n}^{2} /(8 \pi)=B_{n}^{2} / 8 R_{n}^{2}=\psi_{n}^{2} / 8$, can thus be determined. Other quantities of interest are the Alfvén velocity $v_{A, n}=B_{n} / \sqrt{4 \pi \rho}$, the number of islands per unit area $N_{n}$, and $\mathcal{E}_{n}=\epsilon_{n} N_{n}$, the total magnetic energy density of the system.

Island merger changes the above quantities [26,27]. First, the coalescence of two identical islands should conserve mass (and hence area, due to incompressibility): Two islands of radius $R_{n}$ result in an island of radius $R_{n+1}=\sqrt{2} R_{n}$. Second, the magnetic flux should remain constant: $\psi_{n+1}=\psi_{n}$. The number density of islands $N$ halves at each stage; the evolution of other quantities can be determined from the conservation rules above, e.g., $B_{n}$ and $v_{A, n}$ both decrease by $\sqrt{2}$.

To transition from this discrete description to a continuous time evolution, the lifetime for each island generation needs to be computed. We consider coalescence to be a two-stage 
process: an initial island approach, resulting from Lorentz attraction, proceeding at roughly the Alfvénic rate; and the subsequent reconnection of the two islands, taken to be much slower and thus dominating the overall merger duration. We therefore express the merger time for $n$th generation islands as $\tau_{n} \simeq \beta_{\text {rec }, n}^{-1} R_{n} / v_{A, n}$, where $\beta_{\text {rec }, n} \ll 1$ is the dimensionless reconnection rate.

The main parameter controlling the reconnection regime, and hence $\beta_{\mathrm{rec}, n}$, in resistive MHD is the Lundquist number set by the parameters of the merging islands, $S_{n} \equiv R_{n} v_{A, n} / \eta$, where $\eta$ is the (constant) magnetic diffusivity. In particular, if $S_{n} \lesssim 10^{4}$, reconnection proceeds in the Sweet-Parker (SP) regime $[30,31]$ with $\beta_{\mathrm{rec}, n} \simeq S_{n}^{-1 / 2}$; if, instead, $S_{n} \gtrsim$ $10^{4}$, then reconnection proceeds in the plasmoid-dominated regime [32-40] with $\beta_{\text {rec }, n} \simeq 0.01$. Importantly, since $S_{n}=$ $R_{n} v_{A, n} / \eta \propto R_{n} B_{n} \propto \psi_{n}$, which is preserved during mergers, we see that $S_{n}$, and thus $\beta_{\text {rec, } n}$, remain unchanged throughout the evolution $\left(S_{n}=S_{0}\right)$. This nontrivial result implies that which reconnection regime governs the island mergers is set by the initial conditions [41].

From the recursive relations for $R_{n}, \psi_{n}$, and $\beta_{\text {rec }, n}$, we find

$$
\begin{aligned}
& \psi_{n}=\psi_{0}, \quad R_{n}=2^{n / 2} R_{0}, \quad B_{n}=2^{-n / 2} B_{0}, \\
& \mathcal{E}_{n}=2^{-n} \mathcal{E}_{0}, \quad N_{n}=2^{-n} N_{0}, \quad \tau_{n}=2^{n} \tau_{0} .
\end{aligned}
$$

The time taken to reach the $n$th generation is

$$
t_{n}=\sum_{k=0}^{n-1} \tau_{k}=\tau_{0} \sum_{k=0}^{n-1} 2^{k} \approx \tau_{0} 2^{n}, \quad n \gg 1 .
$$

Thus, the relationship between time and island generation $n$ is $t_{n}=\tau_{0} \tilde{t}=\tau_{0} 2^{n}$, where $\tilde{t} \equiv t_{n} / \tau_{0}$. This allows us to eliminate the index $n$ and obtain explicit, continuous time dependencies,

$$
\begin{aligned}
& k=k_{0} \tilde{t}^{-1 / 2}, \quad B=B_{0} \tilde{t}^{-1 / 2}, \\
& \mathcal{E}=\mathcal{E}_{0} \tilde{t}^{-1}, \quad N=N_{0} \tilde{t}^{-1}, \quad \psi=\psi_{0},
\end{aligned}
$$

where $k \equiv 2 \pi / R$.

An alternative derivation of the scaling $B \sim t^{-1 / 2}$, Eq. (3), is obtained by expressing the time evolution of magnetic energy as $d B^{2} / d t \sim B^{2} / \tau_{\text {rec }}$, where $\tau_{\text {rec }}=\beta_{\text {rec }}^{-1} R / v_{A}$ is the reconnection time. The constancy both of the magnetic flux, $\psi=$ $B R$, and of the reconnection rate $\beta_{\text {rec }}$ then implies that $\tau_{\text {rec }} \propto$ $B^{-2}$ and, therefore, $B \sim t^{-1 / 2}$. Interestingly, the same scaling is obtained if we replace $\tau_{\text {rec }}$ with $\tau_{A}=R / v_{A}$ as the characteristic timescale for magnetic energy evolution [3,11,14]. Note, however, that this happens only because of the constancy of $\beta_{\text {rec }}$ that we have derived, causing the reconnection timescale to track the Alfvénic timescale. Physically, the mechanism that dissipates magnetic energy is reconnection, and that is thus what sets its timescale.

The growing length scale and decreasing field strength, Eq. (3), can also be interpreted from the perspective of dynamical renormalization. For an arbitrary scaling factor $l$, Eq. (3) is equivalent to the transformation

$$
k \rightarrow l^{-1} k, \quad \tilde{t} \rightarrow l^{2} \tilde{t}, \quad B \rightarrow l^{-1} B .
$$

It is a confirmation of our dynamical model that these relations are consistent with the general self-similar properties of the (unforced) MHD equations [15,42]; what we have shown, however, is that a physical process exists that enables such a rescaling.

Magnetic spectrum. The evolution of the system which we have just described is not, in fact, characterized by a single scale $\left(k_{\text {isl }}\right)$ : The current sheets (of transverse scale $k_{\mathrm{CS}}$ ) which form during coalescence result in a wide magnetic energy Fourier spectrum, $k_{\text {isl }}<k<k_{\mathrm{CS}}$, where, for SP reconnection, $k_{\text {isl }} / k_{\mathrm{CS}}=S_{0}^{-1 / 2}$ (for $S_{0}<10^{4}$ ). Islands and sheets evolve together $\left[k_{\mathrm{CS}}(t) \propto k_{\text {isl }}(t)\right.$ since $S_{0}=$ const], so, importantly, this entire scale range evolves on the same timescale. Therefore, the magnetic power spectrum $U(k, \tilde{t})$ in this scale range,

$$
U(k, \tilde{t}) \equiv \frac{1}{8 \pi} \frac{2 \pi k}{(2 \pi)^{2}} \int d^{2} r e^{i \mathbf{k} \cdot \mathbf{r}}\langle\mathbf{B}(\mathbf{x}, \tilde{t}) \cdot \mathbf{B}(\mathbf{x}+\mathbf{r}, \tilde{t})\rangle,
$$

transforms as $U\left(k / l, l^{2} \tilde{t}\right)=l^{-1} U(k, \tilde{t})$, according to Eq. (5). The spectra at different times are thus related by the scaling factor $l$, with a self-similar solution $[15,42]$,

$$
U(k, \tilde{t})=\tilde{t}^{-1 / 2} \bar{U}\left(k \tilde{t}^{1 / 2}\right),
$$

where $\bar{U}$ is a scaling function of the variable $k \tilde{t}^{1 / 2}$

In the particular case of a power-law spectrum, $\bar{U}\left(k \tilde{t}^{1 / 2}\right) \propto$ $\left(k \tilde{t}^{1 / 2}\right)^{-\gamma}$, the solution is

$$
U(k, \tilde{t}) \propto \tilde{t}^{-\alpha} k^{-\gamma},
$$

where $2 \alpha=\gamma+1$. In our system, the sharp magnetic field reversals at the current sheets are expected to lead to $\gamma=$ 2 [43] (i.e., a $k^{-2}$ spectrum in the range $k_{\text {isl }}<k<k_{\mathrm{CS}}$ ), and thus $\alpha=3 / 2$. The decay of energy density at any fixed wave number should then scale as $U_{k}(\tilde{t}) \propto \tilde{t}^{-3 / 2}$.

Numerical study. To test the above results, we conduct direct numerical simulations (DNS), using the pseudospectral code VIRIATO [44], of the two-dimensional (incompressible) reduced-MHD equations [45-48]: $\partial_{t} \psi+\mathbf{v}_{\perp} \cdot \nabla_{\perp} \psi=$ $\eta \nabla_{\perp}^{2} \psi ; \partial_{t} \omega+\mathbf{v}_{\perp} \cdot \nabla_{\perp} \omega-\mathbf{B}_{\perp} \cdot \nabla_{\perp} j=v \nabla_{\perp}^{2} \omega$, where the total magnetic field is $\mathbf{B}=B_{z} \hat{\mathbf{z}}+\mathbf{B}_{\perp}$, with $B_{\perp}=|\hat{\mathbf{z}} \times \nabla \psi| \ll$ $B_{z}$, and the vorticity is $\omega=\hat{\mathbf{z}} \cdot\left(\nabla \times \mathbf{v}_{\perp}\right)$. The viscosity $v$ is taken equal to the magnetic diffusivity $\eta$ in all simulations. In what follows, quantities are given in dimensionless form. The domain is a periodic square box with sides of length $L=2 \pi$. The initial equilibrium is described by the stream function $\phi(x, y)=0$ and the magnetic flux function $\psi(x, y)=$ $\psi_{0} \cos \left(k_{0} x\right) \cos \left(k_{0} y\right)$, yielding a $2 k_{0} \times 2 k_{0}$ static array of magnetic islands with opposite polarities (Fig. 1, left panel). In all runs we choose $k_{0}=8$, and thus $R_{0}=L / 4 k_{0}=\pi / 16$. We further set $\psi_{0} k_{0}=1$, implying $B_{0} \equiv \psi_{0} / R_{0}=2 / \pi$. This initial equilibrium is perturbed by small-amplitude, spatially random noise. We perform a series of runs for different values of the initial island-scale Lundquist number $S_{0} \equiv R_{0} v_{A, 0} / \eta \in$ $\{125,179,250,417,1250,1786\}$. We use $8192^{2}$ grid points for $S_{0}=1786 ; 4096^{2}$ for $S_{0}=1250,417$; and $2048^{2}$ for $S_{0}=$ $250,179,125$. The widths of initial (SP) current sheets are resolved with three or four grid points in all cases. Since $S_{0}<10^{4}$ in all runs, reconnection should proceed in the SP regime and no (secondary) plasmoids are expected to arise; visual inspection of our simulations confirms this.

Figure 1 shows the configuration of the system with $S_{0}=$ 1786 at different times. As expected, island mergers lead to the progressive formation of ever larger structures. 


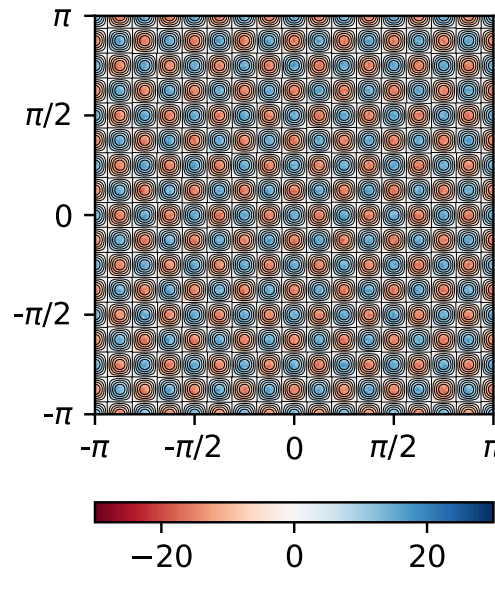

(a)

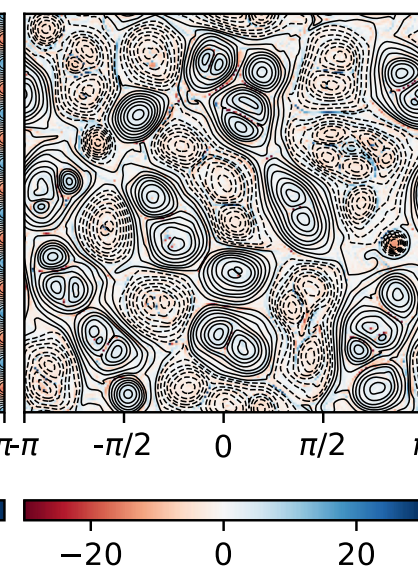

(b)

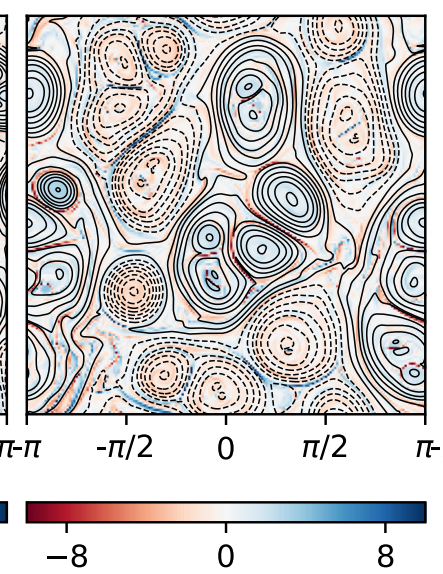

(c)

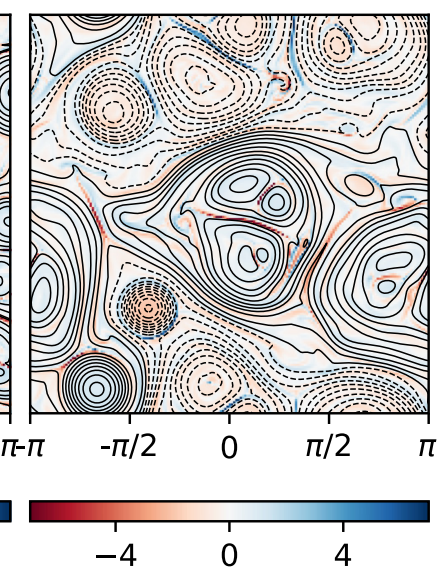

(d)

FIG. 1. Current density (colors) and magnetic flux (contours) at (a) $t=0$, (b) $t=20$, (c) $t=40$, and (d) $t=80$ for the run with $S_{0}=1786$.

In Fig. 2 we plot the time evolution of the total magnetic energy $\mathcal{E}$ for all values of $S_{0}$. After an initial transient period (represented by a time offset $t_{0}$; for $S_{0}=1786$ it is $t_{0}=4$ [49]) the system enters a prolonged stage of self-similar evolution with power-law-in-time behavior; other quantities, such as the number of islands [50] $N(t)$ or the spatial maximum of the flux $\psi_{\max }(t)$, behave similarly. We fit these data to functions of the form $\left(t-t_{0}\right)^{\lambda}$. The measured power-law indices $\lambda_{\mathcal{E}}, \lambda_{N}$, and $\lambda_{\psi}$ converge to the predictions of our hierarchical model, Eq. (4), as $S_{0}$ increases, as shown in Fig. 3. This suggests that our model captures the basic dynamics of the system.

Additionally, Fig. 2 clearly demonstrates that the characteristic timescale for the magnetic energy evolution is the reconnection time $\tau_{0}$. This is evidenced by the approximate collapse of all high $S_{0}$ curves in the main plot, where time is normalized to $\tau_{0}$, but not in the inset figure, where time is in code units.

Figure 4 (top panel) shows the magnetic spectrum $U(k, t)$ at different moments of time for the $S_{0}=1786$ run. As is visually intuited from Fig. 1, we observe that the peak of the

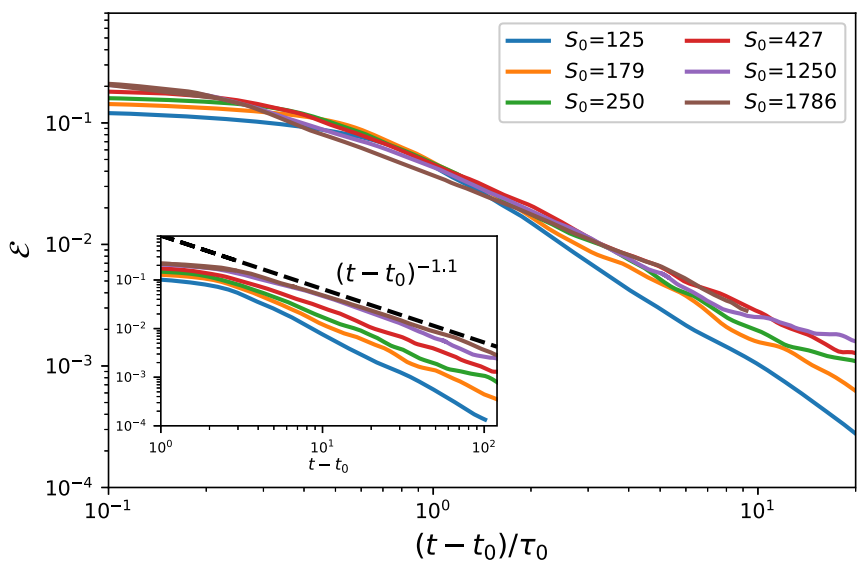

FIG. 2. Time evolution of total magnetic energy $(\mathcal{E})$ for all values of $S_{0}$. Time axis in the main figure is normalized to the reconnection timescale $\tau_{0}=S_{0}^{1 / 2}\left(\pi^{2} / 32\right)$ (in code units). The inset figure shows same data vs time in code units. The power-law fit for $S_{0}=1786$ is indicated for reference. spectrum moves to larger scales, while retaining an overall similar shape. To the right of the peak, these spectra exhibit power-law behavior (an inertial range), with a slope $\gamma \approx 2$ (in agreement with Refs. [51,52]). We think that this index is due to the presence of thin current sheets [43]; indeed, a $k^{-2}$ spectrum forms even before any coalescence has taken place, and thus it cannot be due to the magnetic island distribution. The kinetic energy spectrum (not shown) exhibits a peak at roughly the same wave number as the magnetic energy, but follows a shallower power law that we tentatively identify with $k^{-1}$. While we do not have an explanation for this spectrum, we observe that it being flatter is consistent with the notion that kinetic energy in the current sheets is dominated by the (Alfvénic) outflows (whose spatial profile [53] yields a flat spectrum), plus background flows on the scale of the dominant islands [54]. The total energy, however, shows a spectrum consistent with $k^{-5 / 3}$. We note that our system remains magnetically dominated at all times and at all scales, unlike more typical turbulent systems, where one usually finds rough equipartition between kinetic and magnetic energies.

The self-similarity of the magnetic spectra is clearly demonstrated in the bottom panel of Fig. 4, where we normalize the spectra to their respective maximum values at each moment of time $U_{\max }(t)$ and the wave numbers to the values

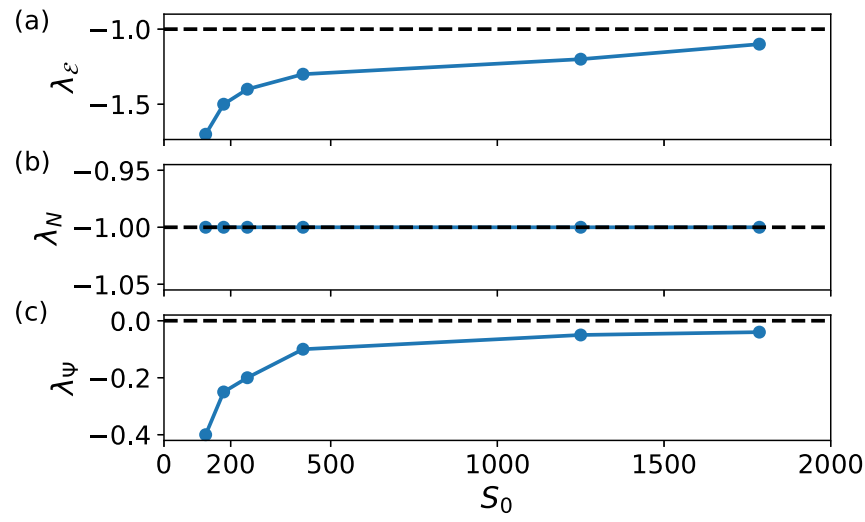

FIG. 3. Power-law exponents as functions of $S_{0}$. Dashed lines represent the predictions of our model, Eq. (4). 
(a)

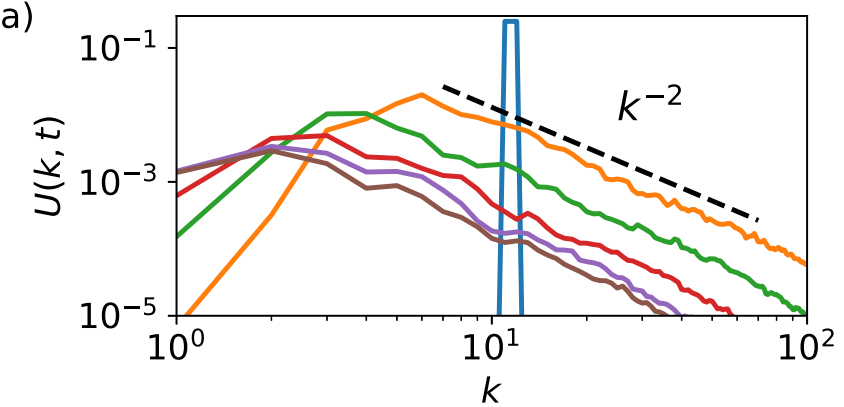

(b)

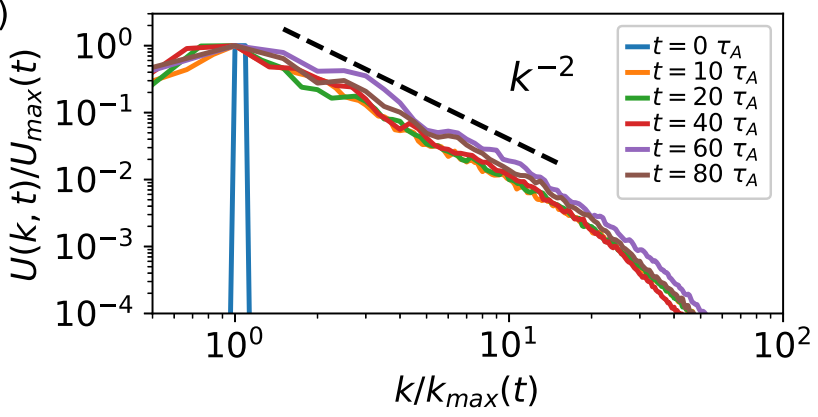

FIG. 4. (a) Raw and (b) normalized magnetic power spectra, for $S_{0}=1786$. A $k^{-2}$ slope is shown for reference.

$k_{\max }(t)$ at which $U_{\max }(t)$ are attained. As seen, all curves essentially collapse onto the same distribution, implying that $U(k, t)=U_{\max }(t) \bar{U}\left(k / k_{\max }\right)$.

We also observe that $k_{\max }$ and $U_{\max }$ are roughly powerlaw functions of time $k_{\max } \propto \Delta t^{-\beta}$ and $U_{\max } \propto \Delta t^{-\theta}$, where $\Delta t \equiv t-t_{0}$, as shown in the top two panels of Fig. 5 . Hence $U(k, t)$ can be expressed as $U(k, t) \propto \Delta t^{-\theta} \bar{U}\left(k \Delta t^{\beta}\right)$, where $\bar{U}\left(k / k_{\max }\right)$ becomes a universal scaling function of the variable $k \Delta t^{\beta}$, consistent with Eq. (7). As noted above, the spectra exhibit an inertial range $\left[k>k_{\max }(t)\right]$ with a power-law dependence on $k: U \propto k^{-\gamma}$ (Fig. 4, top panel). Therefore, in the inertial range, we have the power-law

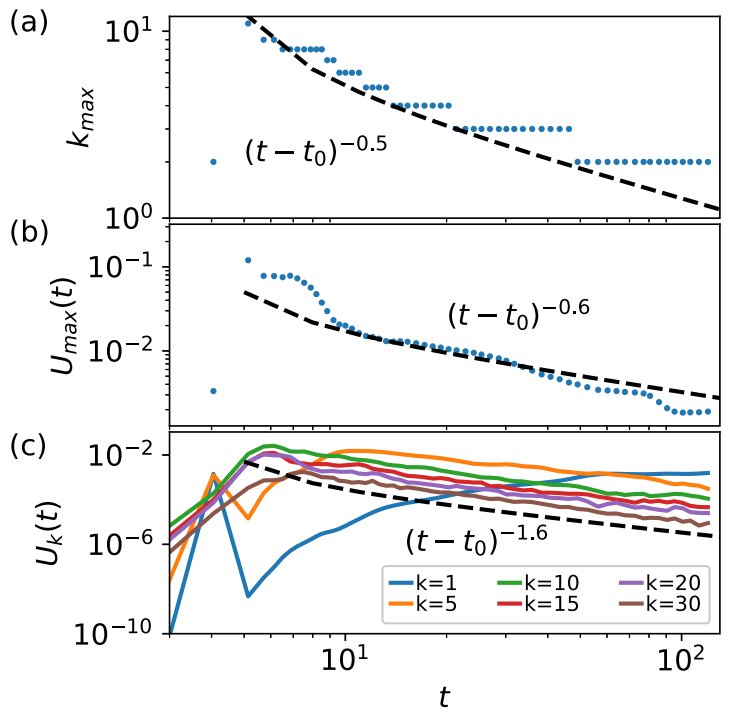

FIG. 5. Time evolution of (a) $k_{\max }$, (b) $U_{\max }$, and (c) $U_{k}$ (for selected values of $k$ ), for $S_{0}=1786$.
TABLE I. Variation of exponents with initial Lundquist number $S_{0}$, compared with the prediction from the hierarchical model.

\begin{tabular}{lcccc}
\hline \hline$S_{0}$ & $\beta$ & $\theta$ & $\alpha$ & $\gamma$ \\
\hline 125 & 0.5 & 1.5 & 3.0 & 3.5 \\
179 & 0.6 & 1.3 & 2.1 & 2.9 \\
250 & 0.6 & 1.3 & 2.0 & 2.1 \\
417 & 0.6 & 1.2 & 1.9 & 2.1 \\
1250 & 0.5 & 1.1 & 1.8 & 2.0 \\
1786 & 0.5 & 0.6 & 1.6 & 2.0 \\
Hyperdissipation & 0.5 & 0.5 & 1.5 & 2.0 \\
Theory & 0.5 & 0.5 & 1.5 & 2.0 \\
\hline \hline
\end{tabular}

scaling function, $\bar{U}\left(k \Delta t^{\beta}\right) \propto\left(k \Delta t^{\beta}\right)^{-\gamma}$, and the power-law time dependence of magnetic spectral energy density at any given $k, U_{k}(t) \propto \Delta t^{-\alpha}$ (Fig. 5, bottom panel), leading to the general expression for the spectrum, $U(k, t) \propto k^{-\gamma} \Delta t^{-\alpha}$, where $\alpha=\gamma \beta+\theta$ (see also Ref. [52]).

The measured values of all indices, as well as the relation between $\alpha$ and $\gamma$, can be compared with our model [Eqs. (7) and (8)]. The results are summarized in Table I, where we also include a $4096^{2}$ simulation performed with hyperdissipation. The exponents approach our theoretical predictions as $S_{0}$ increases.

Lastly, we have performed one run $\left(\eta=10^{-4}, 4096^{2}\right.$ resolution) where the initial condition is instead a Gaussianrandom magnetic field, with a spectrum narrowly peaked around $k_{0}=8$. The power-law exponents obtained in this run are close to those in the run with same $\eta$ but with the periodic-island initial configuration, showing the generality of our reconnection-based hierarchical model.

Conclusions. We have introduced a solvable analytic model to describe the inverse transfer of magnetic energy arising from the hierarchical merger of magnetic islands via magnetic reconnection. We have also carried out direct numerical simulations which show good agreement with the predictions of the model, thereby identifying reconnection as the mechanism that sets the properties (including, importantly, the timescale) of such inverse energy transfer. These results may provide a theoretical understanding of the inverse magnetic energy transfer in nonhelical turbulence observed in recent numerical simulations $[51,52,55]$. More generally, the notion of reconnection as the enabler of inverse energy transfer may be of broad applicability. For example, it may pave the way for understanding the long-term evolution of kineticscale seed magnetic fields, a longstanding problem in plasma astrophysics with direct implications to GRBs and galactic magnetogenesis.

Acknowledgments. This work was supported by NSF CAREER Award No. 1654168 (M.Z. and N.F.L.), NSFDOE Partnership in Basic Plasma Science and Engineering Award No. DE-SC0016215 (P.B.), and NSF Grants No. AST1411879 and No. AST-1806084 and NASA ATP Grants No. NNX16AB28G and No. NNX17AK57G (D.A.U.). D.A.U. gratefully acknowledges the hospitality of the Institute for Advanced Study and the support from the Ambrose Monell Foundation. The authors thank James A. Klimchuk, Giovanni Lapenta, Alexander A. Schekochihin, and Liujun Zou for 
insightful discussions. The simulations presented in this paper were performed on the MIT-PSFC partition of the Engaging cluster at the MGHPCC facility, funded by DOE Award No. DE-FG02-91-ER54109.
[1] R. M. Kulsrud and E. G. Zweibel, Rep. Prog. Phys. 71, 046901 (2008).

[2] E. S. Weibel, Phys. Rev. Lett. 2, 83 (1959).

[3] A. Gruzinov, Astrophys. J. Lett. 563, L15 (2001).

[4] M. V. Medvedev and A. Loeb, Astrophys. J. 526, 697 (1999).

[5] W. B. Manchester IV, T. I. Gombosi, I. Roussev, D. L. De Zeeuw, I. Sokolov, K. G. Powell, G. Tóth, and M. Opher, J. Geophys. Res.: Space Phys. 109, A02107 (2004).

[6] J. A. Klimchuk, S. Patsourakos, and P. J. Cargill, Astrophys. J. 682, 1351 (2008).

[7] O. Khabarova, G. Zank, G. Li, J. Le Roux, G. Webb, A. Dosch, and O. Malandraki, Astrophys. J. 808, 181 (2015).

[8] J. F. Drake, M. Swisdak, and R. Fermo, Astrophys. J. Lett. 763, L5 (2012).

[9] R. H. Kraichnan and D. Montgomery, Rep. Prog. Phys. 43, 547 (1980).

[10] W. Matthaeus and S. L. Lamkin, Phys. Fluids 29, 2513 (1986).

[11] D. Biskamp and H. Welter, Phys. Fluids B 1, 1964 (1989).

[12] D. Biskamp and U. Bremer, Phys. Rev. Lett. 72, 3819 (1994).

[13] D. Biskamp and W.-C. Müller, Phys. Rev. Lett. 83, 2195 (1999).

[14] D. Biskamp and E. Schwarz, Phys. Plasmas 8, 3282 (2001).

[15] P. Olesen, Phys. Lett. B 398, 321 (1997).

[16] S. Galtier, H. Politano, and A. Pouquet, Phys. Rev. Lett. 79, 2807 (1997).

[17] M. Hossain, P. C. Gray, D. H. Pontius, Jr., W. H. Matthaeus, and S. Oughton, Phys. Fluids 7, 2886 (1995).

[18] M. Christensson, M. Hindmarsh, and A. Brandenburg, Phys. Rev. E 64, 056405 (2001).

[19] D. Fyfe and D. Montgomery, J. Plasma Phys. 16, 181 (1976).

[20] A. Pouquet, J. Fluid Mech. 88, 1 (1978).

[21] U. Frisch, A. Pouquet, J. LÉOrat, and A. Mazure, J. Fluid Mech. 68, 769 (1975).

[22] A. Pouquet and G. S. Patterson, J. Fluid Mech. 85, 305 (1978).

[23] M. V. Medvedev, M. Fiore, R. A. Fonseca, L. O. Silva, and W. B. Mori, Astrophys. J. Lett. 618, L75 (2004).

[24] T. N. Kato, Phys. Plasmas 12, 080705 (2005).

[25] B. Katz, U. Keshet, and E. Waxman, Astrophys. J. 655, 375 (2007).

[26] R. Fermo, J. Drake, and M. Swisdak, Phys. Plasmas 17, 010702 (2010).

[27] M. Lyutikov, L. Sironi, S. S. Komissarov, and O. Porth, J. Plasma Phys. 83, 635830602 (2017).

[28] R. H. Kraichnan, J. Atmos. Sci. 33, 1521 (1976).

[29] J. M. Finn and P. Kaw, Phys. Fluids 20, 72 (1977).

[30] P. A. Sweet, in Electromagnetic Phenomena in Cosmical Physics, edited by B. Lehnert, IAU Symposium Vol. 6, (Cambridge University Press, Cambridge, U.K., 1958), p. 123.

[31] E. N. Parker, J. Geophys. Res. 62, 509 (1957).

[32] N. F. Loureiro, A. A. Schekochihin, and S. C. Cowley, Phys. Plasmas 14, 100703 (2007).

[33] G. Lapenta, Phys. Rev. Lett. 100, 235001 (2008).

[34] R. Samtaney, N. F. Loureiro, D. A. Uzdensky, A. A. Schekochihin, and S. C. Cowley, Phys. Rev. Lett. 103, 105004 (2009).
[35] A. Bhattacharjee, Y.-M. Huang, H. Yang, and B. Rogers, Phys. Plasmas 16, 112102 (2009).

[36] Y.-M. Huang and A. Bhattacharjee, Phys. Plasmas 17, 062104 (2010).

[37] D. A. Uzdensky, N. F. Loureiro, and A. A. Schekochihin, Phys. Rev. Lett. 105, 235002 (2010).

[38] N. F. Loureiro, R. Samtaney, A. A. Schekochihin, and D. A. Uzdensky, Phys. Plasmas 19, 042303 (2012).

[39] N. F. Loureiro, A. A. Schekochihin, and A. Zocco, Phys. Rev. Lett. 111, 025002 (2013).

[40] N. F. Loureiro and D. A. Uzdensky, Plasma Phys. Controlled Fusion 58, 014021 (2016).

[41] In the collisionless case $\beta_{\text {rec }} \simeq 0.1$ [56] should also remain constant in time, even though the reconnection regime transitions from laminar to plasmoid mediated as $R_{n}$ grows while the ion skin depth $d_{i}$ remains constant [57], though the efficiency of coalescence may be decreased $[58,59]$.

[42] P. Olesen, arXiv:1509.08962.

[43] J. M. Burgers, in Advances in Applied Mechanics, Vol. 1 (Elsevier, Amsterdam, 1948), pp. 171-199.

[44] N. F. Loureiro, W. Dorland, L. Fazendeiro, A. Kanekar, A. Mallet, M. Vilelas, and A. Zocco, Comput. Phys. Commun. 206, 45 (2016).

[45] B. Kadomtsev and O. Pogutse, Sov. Phys. JETP 5, 575 (1973).

[46] H. R. Strauss, Phys. Fluids 19, 134 (1976).

[47] G. P. Zank and W. H. Matthaeus, J. Plasma Phys. 48, 85 (1992).

[48] A. A. Schekochihin, S. C. Cowley, W. Dorland, G. W. Hammett, G. G. Howes, E. Quataert, and T. Tatsuno, Astrophys. J. Suppl. Ser. 182, 310 (2009).

[49] We find that $t_{0}$ increases with $S_{0}$, consistent with the expected scaling of the reconnection rate.

[50] The number of islands is numerically determined by diagnosing the $O$ points and $X$ points of the system, identified with the maximum/minimum and saddle points of $\psi(x, y)$ (e.g., Ref. [60]).

[51] A. Brandenburg, T. Kahniashvili, and A. G. Tevzadze, Phys. Rev. Lett. 114, 075001 (2015).

[52] J. Zrake, Astrophys. J. Lett. 794, L26 (2014).

[53] N. F. Loureiro, A. A. Schekochihin, and D. A. Uzdensky, Phys. Rev. E 87, 013102 (2013).

[54] We also observe in our simulations that kinetic energy decays as $u^{2} \sim t^{-1}$, consistent with $u \sim B$.

[55] J. Reppin and R. Banerjee, Phys. Rev. E 96, 053105 (2017).

[56] P. Cassak, Y.-H. Liu, and M. Shay, J. Plasma Phys. 83, 715830501 (2017).

[57] H. Ji and W. Daughton, Phys. Plasmas 18, 111207 (2011).

[58] H. Karimabadi, J. Dorelli, V. Roytershteyn, W. Daughton, and L. Chacón, Phys. Rev. Lett. 107, 025002 (2011).

[59] A. Stanier, W. Daughton, L. Chacon, H. Karimabadi, J. Ng, Y.-M. Huang, A. Hakim, and A. Bhattacharjee, Phys. Rev. Lett. 115, 175004 (2015).

[60] S. Servidio, W. H. Matthaeus, M. A. Shay, P. A. Cassak, and P. Dmitruk, Phys. Rev. Lett. 102, 115003 (2009). 